\title{
ASSEMBLABILITY CONSTRAINTS IN THE LIMIT ANALYSIS OF 3D MASONRY INTERLOCKING BLOCKS
}

\author{
ELHAM MOUSAVIAN ${ }^{1 *}$, CLAUDIA CASAPULLA ${ }^{1}$ \\ ${ }^{1}$ Department of Structures for Engineering and Architecture (DiSt) \\ University of Napoli Federico II \\ Via Forno vecchio, 36 - 80134 Napoli, Italy \\ e-mail: elham.mousavian@unina.it (*corresponding author) \\ email: casacla@unina.it
}

Keywords: Assembling path detection; limit analysis for orthotropic interfaces; structural infeasibility measurement; sliding infeasibility; interlocking blocks

\begin{abstract}
This paper presents a method to analyses the structural feasibility and assemblability of the masonry assemblages composed of interlocking blocks. Interlocking blocks with projections and depressions on their faces have relatively better structural performance comparing to the conventional blocks with flat faces, during and after the construction. Therefore, they can represent proper alternatives to the conventional blocks for the seismic retrofitting of unreinforced masonry structures. Structural soundness and assemblability of a model are both functions of the interlocking block geometry. The proposed methods enable the designer to adjust the shape of the interlocking blocks, while meeting the structural and assembling requirements. The paper first introduces an extension of the limit analysis to the assemblages with corrugated interlocking interfaces having anisotropic sliding behavior. Then, the work reformulates the extended limit analysis to develop a method to measure the structural infeasibility due to the lack of sliding resistance at the interlocking interfaces. This is called sliding infeasibility and the designer can minimize it during the shape exploration. Finally, an assemblability method is presented to check if the designed interlocking blocks can be assembled on the other blocks in contact. This method is added to the extended limit analysis and the sliding infeasibility measurement method in form of a geometric constraint that prevents modeling of un-assemblable structures.
\end{abstract}

\section{INTRODUCTION}

Recently, an exponential scientific interest can be observed in the investigation of the inplane and out-of-plane behavior of assemblages composed of interlocking blocks. Several studies demonstrate that the static and dynamic load bearing capacities of such assemblages increase when interlocking blocks are used instead of conventional blocks. Many works were carried out to compare the structural performance of different interlocking shapes (corrugated, cross-shaped, etc.) and to find the geometric parameters by which the structural performances are maximized. Liu et al. [1] tested the in-plane capacity of the masonry walls composed of corrugated joints with different cross sections. Totoev [2] and Hossain et al. [3] 
experimentally and numerically investigated the in-plane and out-of-plane behavior of the walls with corrugated joints. The out-of-plane behavior of masonry walls with osteomorphic blocks [4,5] and cross-shaped joints [6] were also studied, together with the more conventional interlocking constraints [7-9]. Fang et al. [10], Sassu et al. [11] and Cipollini et al. [12] carried out several interesting numerical and experimental analyses on the structural behavior of wooden joineries with various shapes. Mousavian and Casapulla [13] extended the limit analysis to the corrugated interfaces and performed the structural feasibility of such assemblages as a function of the number and orientation of the locks, i.e., the projections and depressions keeping the blocks together and preventing them from sliding.

Furthermore, interlocking blocks can increase the stability of such assemblages during the construction phase. This can considerably decrease the needed formwork and improve the quality of the workmanship. Therefore, interlocking blocks are good candidates for the retrofitting of masonry structures, provided that the old damaged or missing conventional blocks can be replaced by them. In fact, the insertion of these blocks may be allowed without mortar, which can be lost during the time due to the chemical, physical, and mechanical degradation. In sum, the use of such blocks can strengthen the restored assemblages by increasing their bending and sliding resistances and improve the constructability during the restoration proeess. To maximize the structural performance and constructability, the optimal shape of each individual interlocking face can be found. The interlocking blocks with the customized shapes can then be manufactured by advanced machines such as CNC mills. This is in the same line of the classical stereotomy, which is a technique to cut each individual block of an assembla improve the thermal [15].

In this framework interlocking blocks with a plug-in has recently y, which is a technique to cut each
pe [14]. Finally, interlocking blocl
hing to optimize the resources for in
y been developed to design asse
locks with rectangular eross section individual analysis methods, already developed for infinite [16] or finite isotropic associative [17-19] or

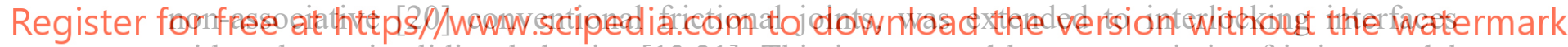
with orthotropic sliding behavior $[13,21]$. This is governed by an associative friction model along the locks and by the lock shear resistance, normally to the locks.

This extended limit analysis is herein reformulated to develop a method to measure the sliding infeasibility (i.e. infeasibility due to violation of sliding constraints) by a numerical value and to introduce assemblability issues. Given the measured sliding infeasibility, the designer can adjust the number and orientation of the locks to minimize this value and reduce the infeasibility in the model. Besides, the lock orientation initially chosen by the designer or adjusted during the shape exploration to reduce the sliding infeasibility, must let the blocks to be assembled or disassembled. When a block can only translate and not rotate, the block in contact with the other blocks is immobilized in some directions, and free-to-move in the others. Choosing the invalid lock orientations, the block can be immobilized (deadlocked) in all directions. Figure 1 shows an example of the deadlocked situation for the central block, which is an invalid model since the blocks cannot be assembled or disassembled.

The developed method follows the Assembly Path Planning (APP) [22] using NonDirectional Blocking Graph (NDBG) developed by Wilson [23]. Given a number of objects in contact, this method describes the directions along which an object cannot move because those directions are blocked by the other objects in contact. When an object is immobilized in 
all directions, the object is so-called un-assemblable. Assembly Path Planning (APP) is one of the major fields of the Assembly Planning AP studies in the manufacturing and industrial engineering, investigating the optimal plans to assemble a whole product composed of smaller parts. A comprehensive review on APP has been carried out by Ghandi and Masehian [24].

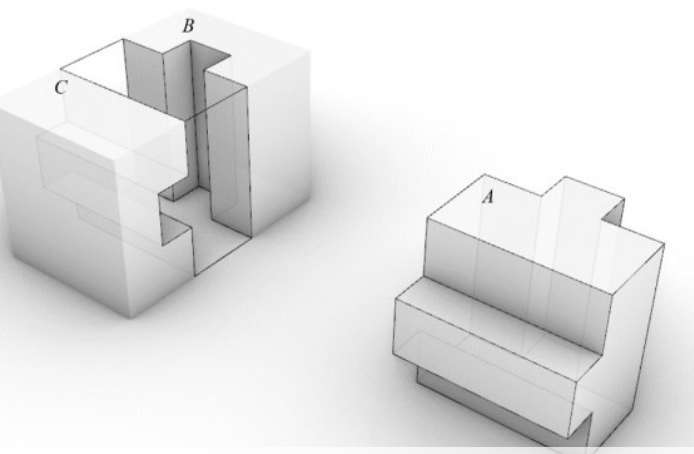

Figure 1: Block $A$ cannot be assembled to Blocks $B$ and $C$ simultaneously.

Given an assembling sequence assigned by the designer, the proposed method finds all the assembling paths along which the block is free to move to be assembled to the other blocks in contact, which were assembled earlier. If no path can be found, the chosen lock orientations for the block faces are detected invalid.

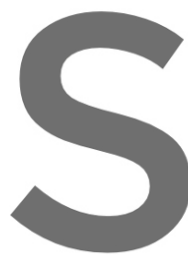

The extended limit introduced in Section the model during the structurally valid mode the performance of the pro
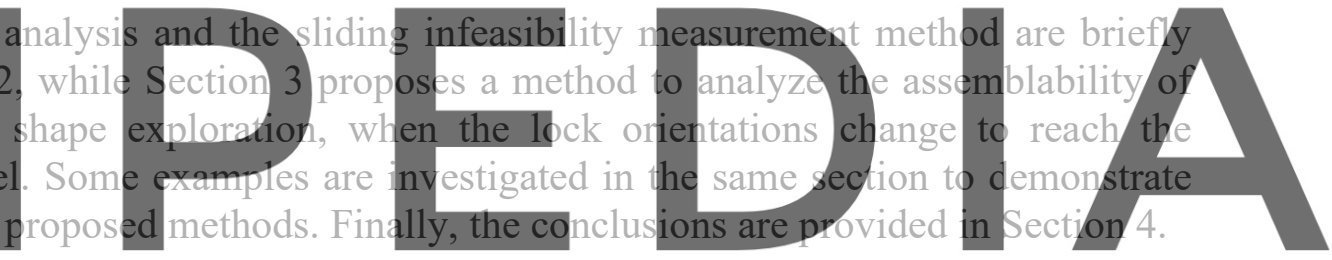

\section{MIETHOD OF SILIDING.INFEASIBIILITY MEASUREMENT}

Register for free at https//Www.scipedia.com to download the version without the watermark Mousavian and Casapulla $[13,21]$ adopted the concave contact model $[17,18]$ to extend

limit analysis to the interlocking corrugated interfaces having locks with rectangular cross section (Figure 2a). Such extended limit analysis is herein applied by using the static approach to find one of the possible stable solutions involving associative friction (equivalent to the Coulomb's friction in the static approach) at the contact interfaces. First, at each interlocking interface, two types of failure planes are determined between the locks of two interlocked blocks (dry joints) and between the locks and the main body of the interlocking blocks (fracture planes) (Figure 2b). The locks and the main body of the blocks are considered rigid enough. Merging the dry joint and fracture plane of a single lock, a number of contact points can be distributed on the lock centerline. Internal forces on these points must equilibrate the external forces applied to the block centroids under the following constraints: a) the normal component $r^{n}$ of each internal force must be in compression, b) the tangential component $r^{t 1}$ along the locks must satisfy the associative friction model, c) the tangential component $r^{t 2}$ normal to the lock must be less than the shear resistance at each contact point. This resistance is a portion $p$ of the total shear resistance $T_{0}$ of the lock, which is considered to be $T_{0}=\left(\tau_{k} s b\right), s$ and $b$ being the lock thickness and length, respectively, and $\tau_{k}$ the material shear strength. The two options for the contact point distribution and shear resistance 
definition sketched in Figure $2 \mathrm{c}$ are chosen among others [13,21] since they provide the closest shear-torsion behavior to the actual one obtained by a literature experimental test [25].

Solving the linear equilibrium equations under the compression, frictional and shear constraints, the internal forces at the contact points, assumed as variables, are found.The mathematical problem, solved by MATLAB's lsqlin using least square optimization, is formulated as follows:

$$
\left\{\begin{array}{lr}
C_{e q} \cdot \vec{r}+\vec{E}=0 & \text { Equilibrium equation } \\
\text { subjected to: } & \\
\overrightarrow{r^{n}} \leq 0 & \text { compression constraint } \\
\left|\overrightarrow{r^{t 1}}\right| \leq \mu\left|\overrightarrow{r^{n}}\right| & \text { friction constraint } \\
\left|\overrightarrow{r^{t 2}}\right| \leq \vec{p} T_{0} & \text { shear constraint }
\end{array}\right.
$$

where $C_{e q}$ is the equilibrium coefficient matrix; $\overrightarrow{r n}, \overrightarrow{r t 1}, \overrightarrow{r^{t 2}}$ are the vectors of the normal and tangential components along the locks, and parallel to them, respectively; $\vec{r}$ is a vector of all the components of the internal forces; $\vec{p}$ is a vector of weights related to the shear resistance at the contact points; $\vec{E}$ is a vector of the external forces and moments. When the solver finds no solution (solver's exit flag is greater than one) the model is recognized structurally infeasible.
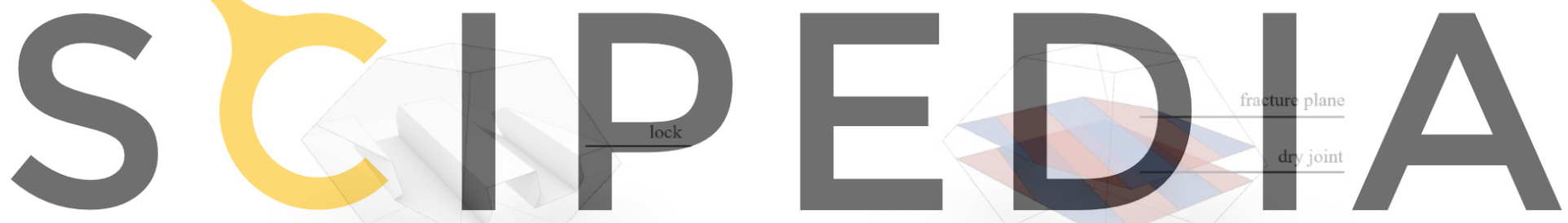

Register for free at https//www.scipedia.com to download the version without the watermark
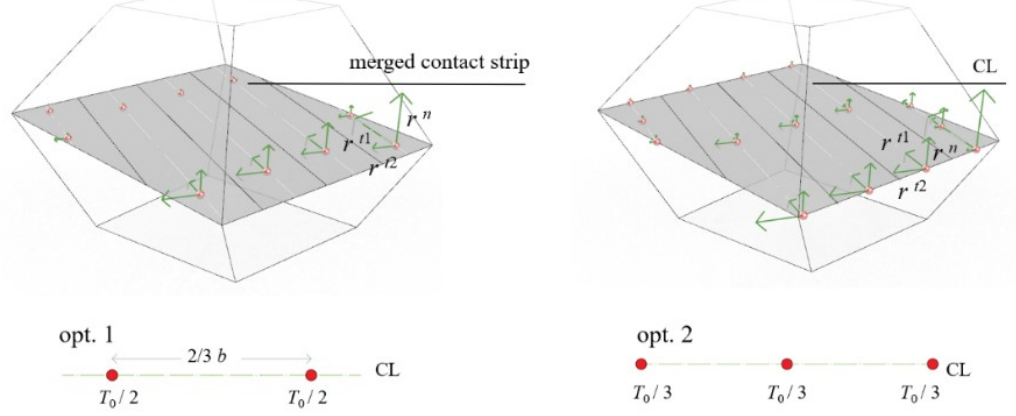

c)

Figure 2: a) Two interlocking blocks with a shared corrugated interface; b) failure planes at the interlocking interface; c) two options for the contact point distribution and shear resistance definition.

The designer can adjust the geometric parameters of the model to remove the structural infeasibility. However, it is usually difficult to guess how to change to parameters to reach a feasible model. If the detected infeasibility can be measured by a numerical value, the 
designer can easily tune the parameters to reduce the measured infeasibility to eventually minimize it.

Whiting et al. [25] proposed a method to quantify the structural infeasibility caused by the tensile forces. Similarly, this work measures the infeasibility due to the tangential forces that violate the sliding (friction and shear) constraints, namely sliding infeasibility. Given the measured sliding infeasibility, the designer can adjust the geometric parameters of the interlocking interfaces to minimize it.

The tangential forces can first get any arbitrary value and then the values that are out of the sliding valid ranges are adopted in the optimization process to measure the sliding infeasibility. In this method, however, the internal and external forces are constrained to be in equilibrium and all the normal forces must be in compression.

The extended limit analysis in Eq. (1) is reformulated to develop this method. To this aim, each individual tangential force $r^{t}$ in the vector of variables is replaced by two new variables $r^{t a}$ and $r^{t b}$ so that: $r^{t}=\left(r^{t a}+r^{t b}\right)$, i.e., $r^{t 1}$ parallel to the locks is replaced by the sum of $r^{t 1 a}$ and $r^{t 1 b}$, and similarly $r^{t 2}$ normal to the locks is replaced by the sum of $r^{t 2 a}$ and $r^{t 2 b}$. While $r$ ${ }^{t 1 a}$ and $r^{t 2 a}$ are respectively constrained to satisfy the friction and shear constraints, $r^{t 1 b}$ and $r$ $2 b$ can take any arbitrary value.

Then, with the objective function that tries to minimize $r^{t 1 b}$ and $r^{t 2 b}$, the optimization problem is posed as follows:
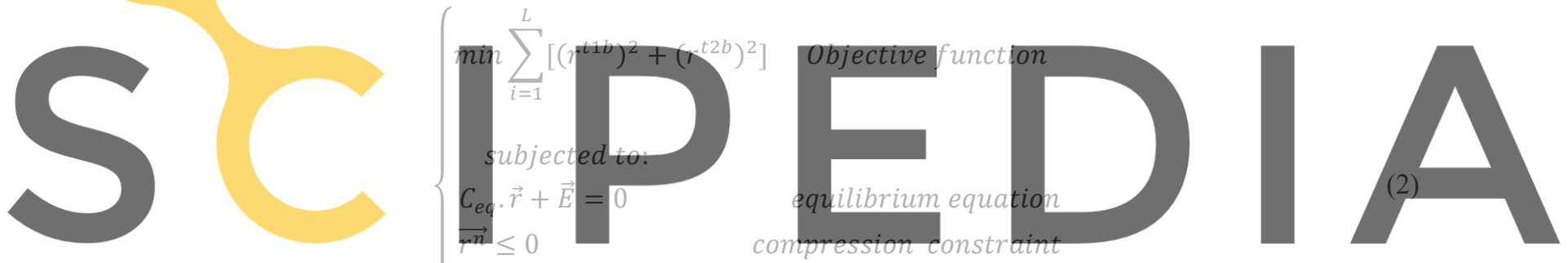

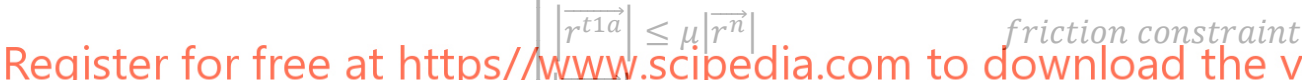
Register for free at https//www.scipedia.com to download the version without the watermark

The resuit of the objective function is defined as the sliding infeasibility measure.

\subsection{Validation}

The extended limit analysis and the sliding infeasibility measurement method have been implemented to develop a plug-in within the grasshopper $(\mathrm{GH})$ editor, using C\# language to model the single layer assemblage with stack bond pattern. The optimization problems are solved by MATLAB solvers which are used as backend. The linear limit analysis and sliding infeasibility measurement problems are solved by MATLAB's lsqlin and fmincon methods, respectively.

To validate the developed plug-in, a semi-circular arch is modeled with $10 \mathrm{~m}$ centerline radius, $1 \mathrm{~m}$ depth, and 40 blocks under their own weight with $1 \mathrm{~N} / \mathrm{m}^{3}$ density. Figure 3 shows the relation between the minimum thickness of an arch with conventional interfaces and the friction coefficient. It can first be observed that the results obtained by the developed plug-in are in good agreement with the results reported by Gilbert et al. [20] and Casapulla et al. [27]. Then, it is evident that the arch with thickness $1.06 \mathrm{~m}$ is structurally infeasible when the 
friction coefficient is less than 0.4. In this case, interlocking blocks with large shear strength can be used to increase the sliding resistance of the interfaces.

Table 1, row 1, reports the sliding feasibility of the arch with $1.06 \mathrm{~m}$ thickness for different lock orientations, when the friction coefficient $\mu$ is 0.33 . The results are obtained by the extended limit analysis of Eq. (2). As expected, when the locks are parallel to the arch plane (lock orientation $\pi / 2$ ), the assemblage is still infeasible. Changing the lock orientation from $\pi / 2$ to $0 \mathrm{rad}$, the sliding resistance of the interfaces increases and eventually the assemblages with lock orientation less than 0.9 rad are recognized feasible.

The second row of Table 1 displays the measured sliding infeasibility of the arch for the different lock orientations. As expected, the infeasibility measure is reduced when the lock orientation changes from $\pi / 2$ to $0 \mathrm{rad}$. Considering that the sliding infeasibility measure of a feasible model must be a number very close to zero, the infeasibility measures less than 0.1 reported in Table 1 may represent the feasible arch.

Table 2 reports the sliding feasibility of the arch with friction coefficient equal to 10, using the extended limit analysis and also the sliding infeasibility measures for the different lock orientations. As expected, all the models can be detected structurally feasible and their measured sliding infeasibility is very close to zero.
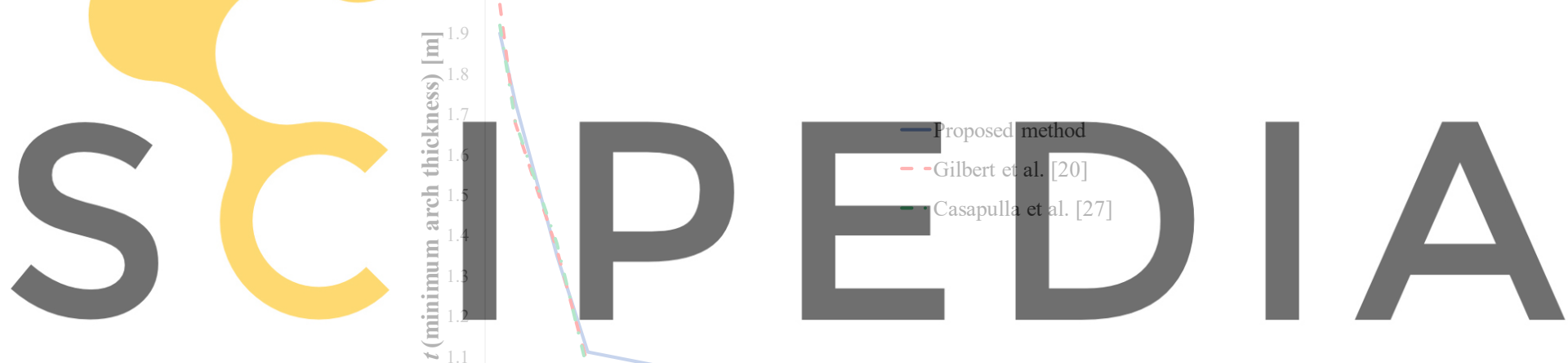

Register for free at https//www.scipedia.com to download the version without the watermark

$\mu$ (friction coefficient)

Figure 3: The thinnest semi-circular arch for different friction coefficients reported by the proposed method, Gilbert et al. [20] and Casapulla et al. [27].

Table 1: Measure of sliding infeasibility for $\mu$ 0.33; f and i stand for feasibility and infeasibility, respectively.

\begin{tabular}{cccccccc}
\hline & $\mu$ lorientation $(\mathrm{rad})$ & 0 & 0.3 & 0.6 & 0.9 & 1.2 & 1.57 \\
\hline limit analysis & 0.33 & $\mathrm{f}$ & $\mathrm{f}$ & $\mathrm{f}$ & $\mathrm{f}$ & $\mathrm{i}$ & $\mathrm{i}$ \\
\hline sliding infeasibility measure & 0.33 & 0.026 & 0.044 & 0.064 & 0.084 & 0.11 & 0.16 \\
\hline
\end{tabular}

Table 2: Measure of sliding infeasibility for $\mu 10$; f and i stand for feasibility and infeasibility, respectively.

\begin{tabular}{cccccccc}
\hline & $\mu$ lorientation $(\mathrm{rad})$ & 0 & 0.3 & 0.6 & 0.9 & 1.2 & 1.57 \\
\hline limit analysis & 10 & $\mathrm{f}$ & $\mathrm{f}$ & $\mathrm{f}$ & $\mathrm{f}$ & $\mathrm{f}$ & $\mathrm{f}$ \\
\hline $\begin{array}{c}\text { sliding infeasibility } \\
\text { measure }\end{array}$ & 10 & $4 \times 10^{-4}$ & $9 \times 10^{-4}$ & 0.001 & 0.002 & 0.007 & 0.01 \\
\hline
\end{tabular}




\section{ASSEMBLABILITY ANALYSIS}

This section introduces a method to check the assemblability/disassemblability of a rigid interlocking block. In this work, a block is only allowed to translate and not to rotate and at every assembling sequence, only one block is added to the assemblage. The concept is to investigate if there is at least one translational path through which the block can be assembled or disassembled. Obviously, when a block can be assembled by translating it along a direction, it can be disassembled along the opposite direction. Here, the algorithm detects the disassemblability paths since the demonstration of the directions where an interlocked block is blocked or free to move is more straight-forward.

When two 2D Objects $A$ and $B$ are placed side by side, as in Figure 4, Object $A$ cannot move along all the directions whose terminal points make circular $\operatorname{arc} c_{A}{ }^{b}$ and Object $B$ is blocked by Object $A$ in all the directions whose terminal points make circular $\operatorname{arc} c_{B}{ }^{b}$. Combining these two circular arcs, the Non-Directional Blocking Graph (NDBG) of the whole assembly is modeled. This graph invented by Wilson [23] has been widely used for the Assembly Path Planning.
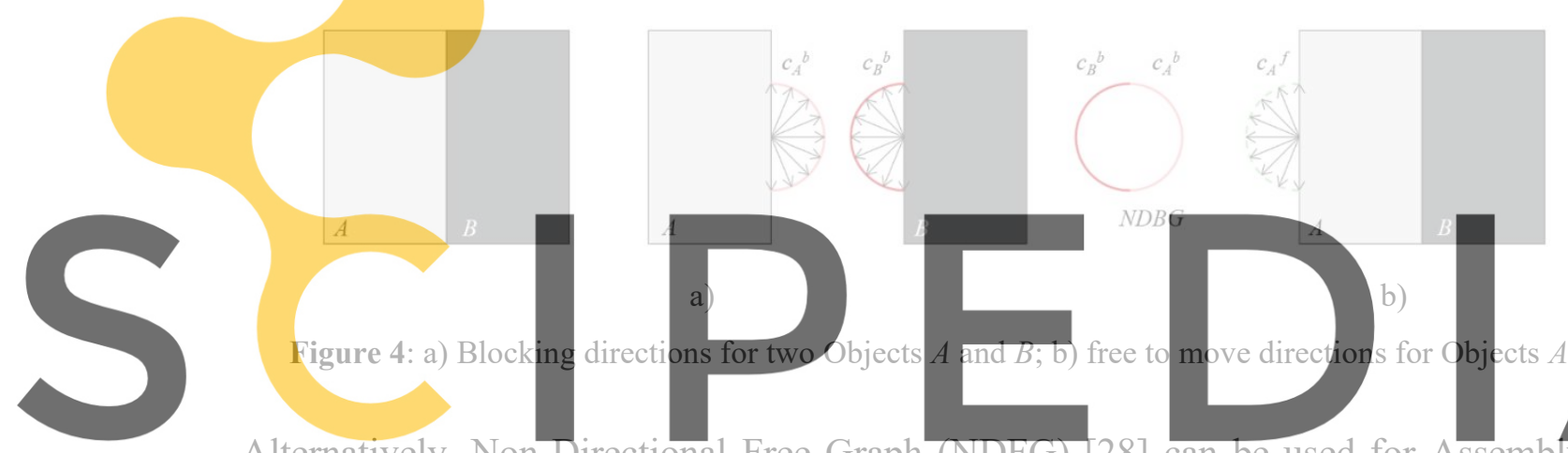

Alternatively, Non-Directional Free Graph (NDFG) [28] can be used for Assembly Path

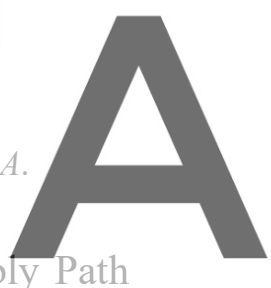
Planning as well, which is a combination of the circular $\operatorname{arcs} c^{f}$, representing the directions

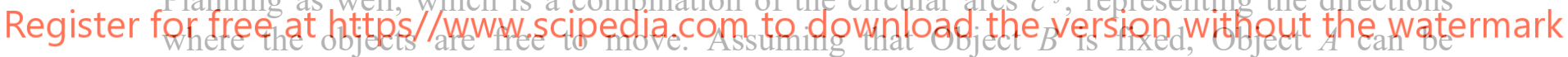
disassembled from Block $B$ only by translating along one of the directions represented by $C_{A}{ }^{f}$.

When Objects $A$ and $B$ have two edges in contact (Figure 5), the circular $\operatorname{arcs} c_{A 1}{ }^{b}$ and $c_{A 2}{ }^{b}$ represent the directions along which Object $A$ is blocked by edges $E_{1}$ and $E_{2}$, respectively. Union of $c_{A 1}{ }^{b}$ and $c_{A 2}{ }^{b}$ makes $c_{A}{ }^{b}$ which represents all the directions along which Object $A$ is blocked by Object $B$. And eventually, subtracting $c_{A}{ }^{b}$ from a circle, all the directions where Object $A$ is free to move is represented by $c_{A}{ }^{f}$.
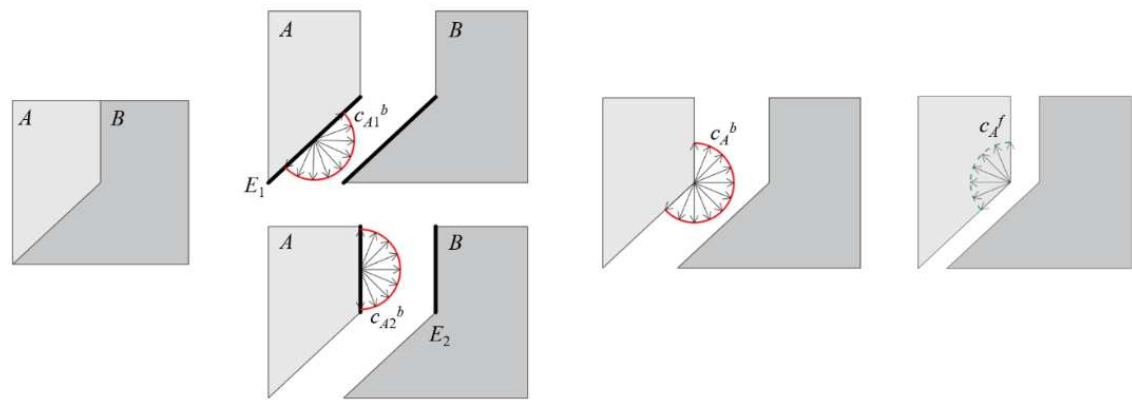

Figure 5: Blocking and free directions for Object $A$, when Objects $A$ and $B$ have two shared edges. 
Similarly, for two 3D Objects $A$ and $B$ with three planar faces in contact (Figure 6), sections of spheres $s_{A 1}{ }^{b}, s_{A 2}{ }^{b}$, and $s_{A 3}{ }^{b}$ demonstrate the directions along which Objects $A$ is blocked by Faces $F_{1}, F_{2}$, and $F_{3}$, respectively. Union of $s_{A 1}{ }^{b}, s_{A 2}{ }^{b}$, and $s_{A 3}{ }^{b}$ makes $s_{A}{ }^{b}$ which displays all the directions along which Object $A$ is blocked by Object $B$. Subtracting $s_{A}{ }^{b}$ from an entire sphere models $s_{A}{ }^{f}$, which demonstrates the directions along which Object $A$ is free to move. When Object $B$ is fixed, the semi-circular arc $s_{A}{ }^{f}$ shows all the valid translational paths through which Object $A$ can be disassembled from Object $B$. It is worth noting that Objects $A$ and $B$ in Figure 6 are interlocking blocks having one lock and increasing the number of the locks, $s_{A}^{f}$ does not change.

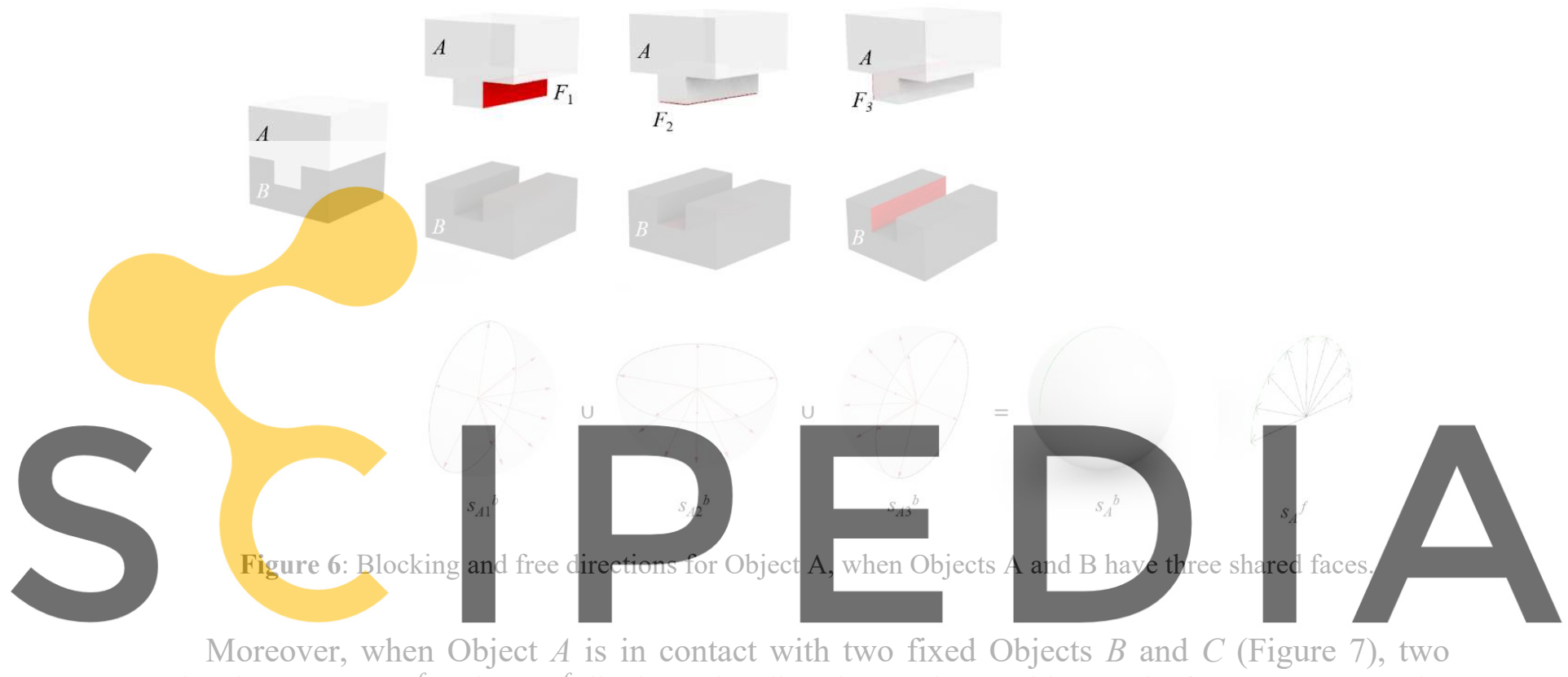

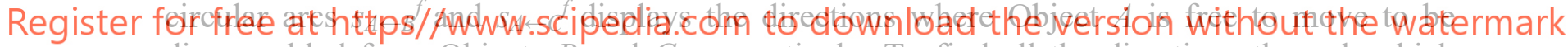
disassembled from Objects $B$ and $C$, respectively. To find all the directions through which Object $A$ can be disassembled from Objects $B$ and $C$ simultaneously, Object $A$ should first be abstracted to its centroid $O$ and then, the arcs $s_{A \leftarrow B}{ }^{f}$ and $s_{A \leftarrow C} C^{f}$ can be modeled with their centers passing through $O$. Intersection of $s_{A \leftarrow B} f$ and $s_{A \leftarrow C} f$ shows the paths along which Object $A$ is free to be disassembled from Objects $B$ and $C$ at the same time. It is evident that Object $A$ can be disassembled from Objects $B$ and $C$ in Figure 7a while it is immobilized in Figure $7 \mathrm{~b}$.

This method is extendable to find the directions through which Object $i$ can be separated from fixed Objects 1 to $i-1$, where objects are enumerated according to the disassembling sequence assigned by the designer. With reference to Figure 8 , arcs $s_{i \leftarrow 1} f$ to $s_{i \leftarrow i-1} f^{f}$ displaying the directions where Object $i$ is free to move to be separated from Objects 1 to $i-1$, respectively, are first modeled with their centers passing through the centroid of Object $i$. Then, the intersection of these arcs displays the valid disassembling path for Object $i$. If the intersection is null (Figure 8a), there is no valid disassembling path, and this shows that the designed model is not assemblable/disassemblable. Therefore, the assemblability constraint can be defined as follows:

$$
\forall \text { Object } i \quad s_{i \leftarrow 1}^{f} \cap s_{i \leftarrow 2}^{f} \cap \ldots \cap s_{i \leftarrow i-1}^{f} \neq \emptyset
$$



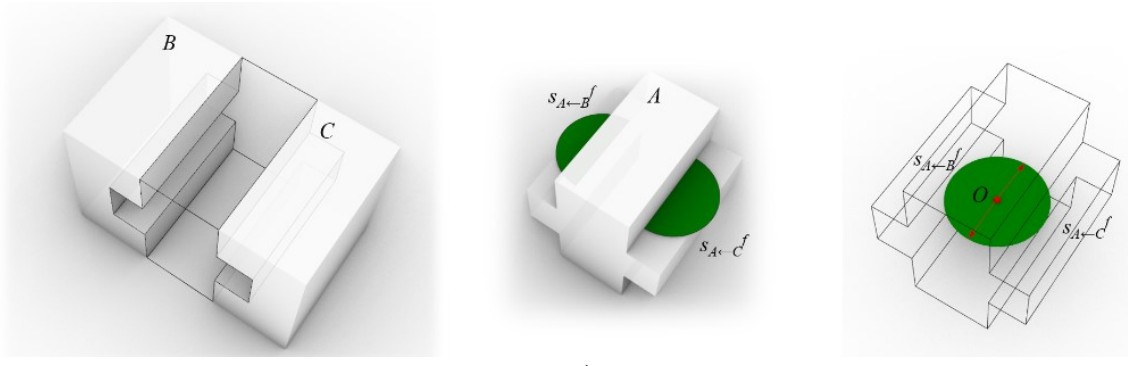

a)
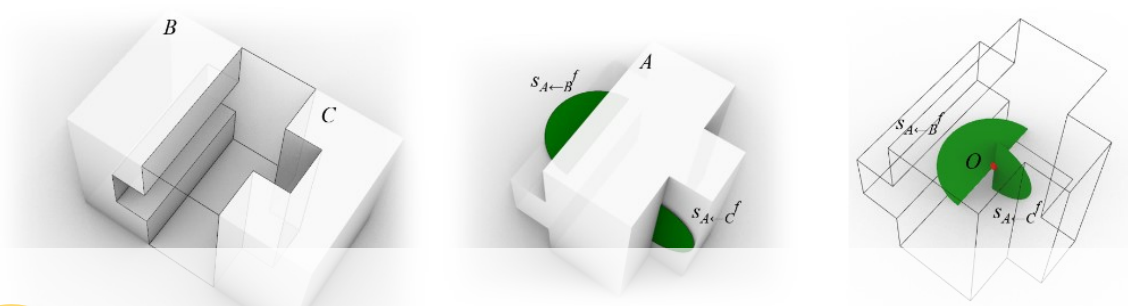

b)

Figure 7: a) Object A is free to move in two directions displayed by two red arrows; b) Object A is immobilized.
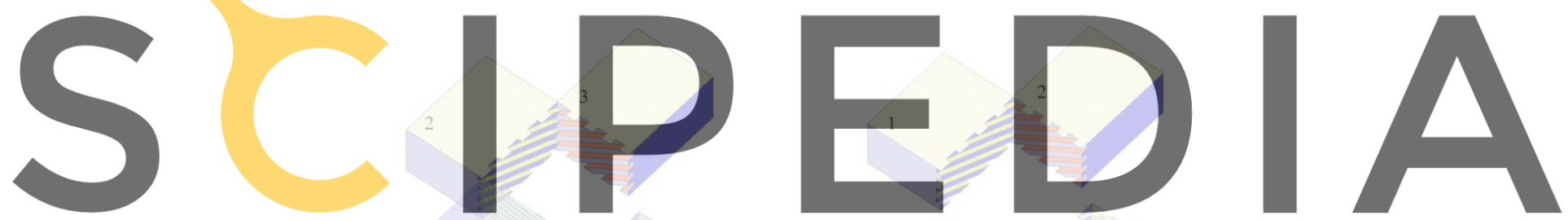

Register for free at https//www.scipedia.com to download the version without the watermark

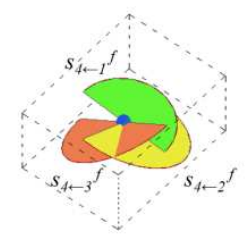

a)

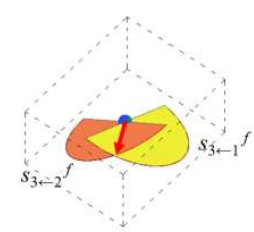

b)

Figure 8: a) Object 4 cannot be disassembled from Objects 1, 2, and 3; b) Object 3 can be disassembled from Objects 1, 2 along the red vector.

Introducing this constraint to both extended limit analysis (Eq. (4)) and sliding infeasibility analysis method (Eq. (5)) prevents modeling of structure whose blocks cannot be assembled, and the two formulations can be expressed as: 


$$
\left\{\begin{array}{lr}
C_{\text {eq }} \cdot \vec{r}+\vec{E}=0 & \text { Equilibrium equation } \\
\text { subjected to: } & \\
\overrightarrow{r^{n}} \leq 0 & \text { compression constraint } \\
\left|\overrightarrow{r^{t 1}}\right| \leq \mu\left|\overrightarrow{r^{n}}\right| & \text { friction constraint } \\
\left|\overrightarrow{r^{t 2}}\right| \leq \vec{p} T_{0} & \text { shear constraint } \\
\forall \text { Object } i \quad s_{i \leftarrow 1}^{f} \cap s_{i \leftarrow 2}^{f} \cap \ldots \cap s_{i \leftarrow i-1}^{f} \neq \emptyset & \text { assemblability constraint }
\end{array}\right.
$$$$
\int \min \sum_{i=1}^{L}\left(r^{t 1 b}\right)^{2}+\left(r^{t 2 b}\right)^{2} \quad \text { objective function }
$$

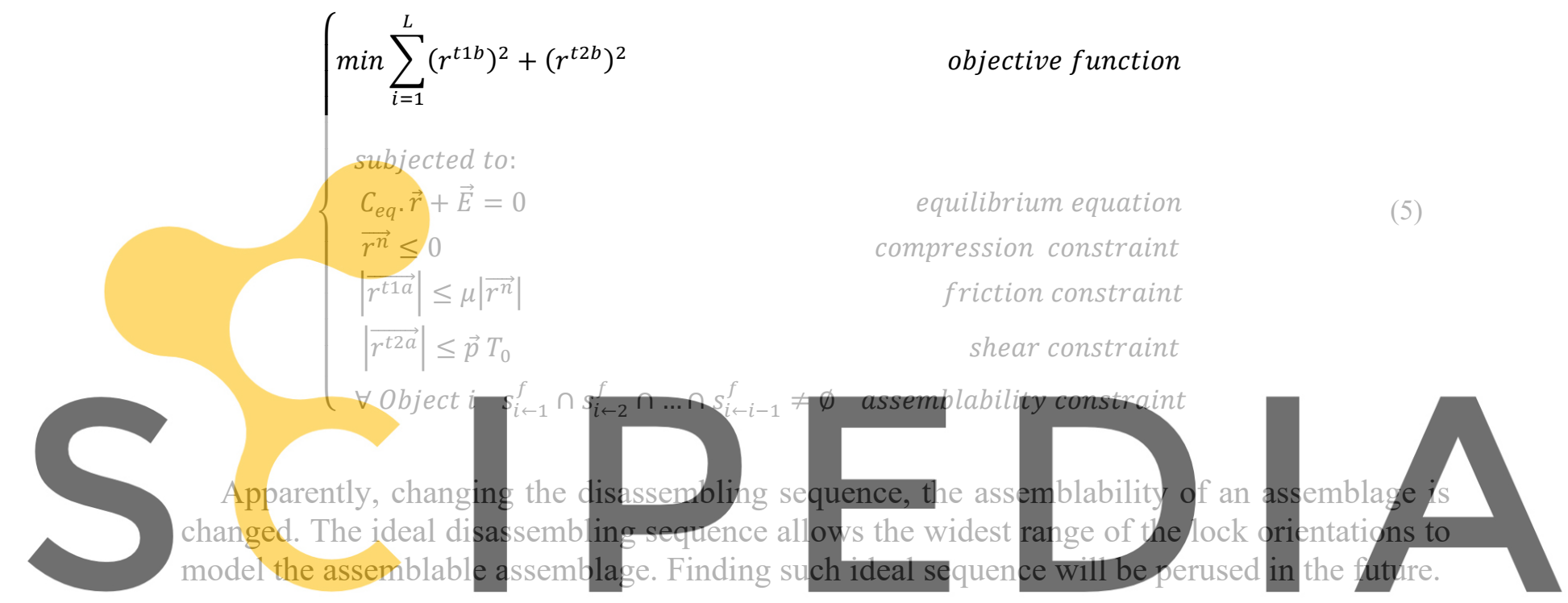

Register for free at https//Www.scipedia.com to download the version without the watermark This work introduced novel methods to measure the sliding feasibility of an assemblage of interlocking blocks and to check the assemblability of the blocks. It first introduced an extension of limit analysis to the interfaces with orthotropic sliding behavior. The extended limit analysis was then reformulated to develop a method to quantify and measure the sliding infeasibility of a model. This helps the designer to minimize the sliding infeasibility easier. Lastly, the assemblability analysis was described as a method to check if the interlocking blocks can be assembled on the other blocks in contact, when they can only be translated to their final positions in the assemblage. This method was integrated with the limit analysis and the sliding infeasibility measurement methods, preventing the blocks of a structurally sound assemblage to be immobilized, i.e., un-assemblable. In future, the work will be extended to find the optimal assembling sequence by which the widest range of the structurally feasible and assemblable lock orientations can be attained.

Acknowledgements. This project has received funding from the European Union's Horizon 2020 research and innovation programme under the Marie Skłodowska-Curie Grant Agreement No. 791235. 


\section{REFERENCES}

[1] Liu, H., Liu, P., Lin, K. and Zhao, S. Cyclic behavior of mortarless brick joints with different interlocking shapes. Materials (2016) 9(3): art. no. 166.

[2] Totoev, Y.Z. Design procedure for semi interlocking masonry. Journal of Civil Engineering and Architecture (2015) 9: 517-525.

[3] Hossain, M. A., Totoev, Y.Z. and Masia, M.J. Friction on Mortar-less Joints in Semi Interlocking Masonry. In: C. Modena et al. (Eds.): Brick and Block Masonry-Trends, Innovations and Challenges, CRC Press/Balkema (2016), pp. 1635-1644.

[4] Dyskin, A.V., Pasternak, E. and Estrin, Y. Mortarless structures based on topological interlocking. Frontiers of Structural and Civil Engineering (2012) 6(2):188-197.

[5] Dyskin, A. V., Estrin, Y. and Pasternak, E. Topological Interlocking Materials. In: Y. Estrin, et al. (Eds.). Architectured Materials in Nature and Engineering, Springer (2019), pp. 23-49.

[6] Ali, M., Gultom, R.J. and Chouw, N. Capacity of innovative interlocking blocks under monotonic loading. Construction and Building Materials (2012) 37:812-821.

[7] Giresini, L. Design Strategy for the Rocking Stability of Horizontally Restrained Masonry Walls. In: M. Papadrakakis and M. Fragiadakis (Eds.): Proceedings of the 6th ECCOMAS Thematic Conference on Computational Methods in Structural Dynamics and Earthquake Engineering (COMPDYN 2017), National Technical University of Athens (2017), pp. 2963-2979.

[8] Giresini, L., Sassu, M. and Sorrentino, L. In situ free-vibration tests on unrestrained and restrained rocking masonry walls. Earthquake Engineering and Structural Dynamics (2018) 47(15):3006-3025.

[9] Giresini, L., Lourenço, P.B., Puppio, M.L., Sassu, M. Rocking and Kinematic Analysis of Two Masonry Church Façades. In: K. Van Balen and E. Verstrynge (Eds.): Structural Analysis of Historical Constructions (SAHC 2016), CRC Press/Balkema (2016), pp. 1190-1196.

[10] Fang, D., Moradei, J., Brütting, J., Fischer, A., Landez, D.K., Shao, B. and Mueller, C. Modern Timber Design Approaches for Traditional Japanese Architecture: Analytical, Experimental, and Numerical Approaches for the Nuki Joint. In: C. Lázaro et al. (Eds.): Form and Force (IASS 2019), CIMNE (2019), pp. 2911-2918.

[11] Sassu, M., De Falco, A., Giresini, L. and Puppio, M. Structural solutions for low-cost bamboo frames: Experimental tests and constructive assessments. Materials (2016) 9(5): art. no. 346.

[12] Cipollini, M., Bonannini, E.,Cinotti, M., Sassu, M. Design, production, and installation of wooden walls for the Japan Pavilion at Expo 2015. Buildings (2016) 6(4): art. no. 43.

[13] Mousavian, E. and Casapulla, C. Structurally informed design of interlocking block assemblages using limit analysis. Journal of Computational Design and Engineering (2020) 7(4):1-21.

[14] Rippmann M., Curry J., Escobedo D. and Block P. Optimising Stone-Cutting Strategies for Freeform Masonry Vaults. In: J.B. Obrębski and R. Tarczewski (Eds.): Proceedings of the International Association for Shell and Spatial Structures (IASS) Symposium (2013), pp. 1-7.

[15] Sassu, M., Stochino, F., Mistretta, F. Assessment method for combined structural and 
energy retrofitting in masonry buildings. Buildings (2017) 7(3): art. no. 71.

[16] Heyman, J. The stone skeleton. International Journal of solids and structures (1966) 2(2):249-279.

[17] Livesley, R.K. Limit analysis of structures formed from rigid blocks. International Journal for Numerical Methods in Engineering (1978) 12(12):1853-1871.

[18] Livesley, R.K. A computational model for the limit analysis of three-dimensional masonry structures. Meccanica (1992) 27(3):161-172.

[19] Casapulla, C. and Maione, A. Modelling the dry-contact interface of rigid blocks under torsion and combined loadings: concavity vs. convexity formulation. International Journal of Non-Linear Mechanics (2018) 99:86-96.

[20] Gilbert, M., Casapulla, C. and Ahmed, H.M. (2006). Limit analysis of masonry block structures with non-associative frictional joints using linear programming. Computers and Structures 84(13-14):873-887.

[21] Mousavian, E. and Casapulla, C. Limit State Approach for Structurally informed Design of Shells Composed of Interlocking Blocks. In: C. Lázaro et al. (Eds.): Form and Force (IASS 2019), CIMNE (2019), pp. 1610-1618.

[22] Canny, J. The complexity of robot motion planning. MIT press (1988).

[23] Wilson, R.H. On Geometric Assembly Planning, PhD thesis No. STAN-CS-92-1416 (1992) Stanford University, Department of Computer Science.

[24] Ghandi, S. and Masehian, E. Review and taxonomies of assembly and disassembly path planning problems and approaches. Computer-Aided Design (2015) 67:58-86.

[25] Casapulla, C. and Portioli F. Experimental tests on the limit states of dry-jointed tuff blocks. Materials and Structures (2016) 49(3):751-767.

[26] Whiting, E., Ochsendorf, J. and Durand, F. Procedural modeling of structurally-sound masonry buildings. ACM Transactions on Graphics (2009) 28(5):112:1-112:9.

[27] Casapulla, C., Mousavian, E. and Zarghani, M. A digital tool to design structurally feasible semi-circular masonry arches composed of interlocking blocks. Computers and Structures (2019) 221:111-126.

[28] Tai, A.S.C. Design for assembly: a computational approach to construct interlocking wooden frames, $\mathrm{PhD}$ thesis (2012) Massachusetts Institute of Technology. 